\title{
FATIGUE ASSESSMENT OF UNIVERSAL CARDAN JOINT BASED ON LABORATORY SPECIMEN TESTS*
}

\author{
Pietro Palma ${ }^{1}$ \\ Gianni Tiussi² \\ Andrea Donadon ${ }^{3}$ \\ Yuri Raffaglio 4 \\ Andrea De Luca ${ }^{5}$ \\ Martin Leitner ${ }^{6}$ \\ Florian Grün ${ }^{7}$ \\ Denis Benasciutti ${ }^{8}$
}

\begin{abstract}
Universal cardan joints are widely used in drivetrains of rolling mills for steel and aluminum making, as well as for non ferrous alloy industry. Working conditions are extremely tough due to high alternating or pulsating rolling torques in the roughing stands and high torques and speeds in the finishing stands. Overloads and lubrication along with oxide and slag are also critical issues that contribute to create severe working conditions. For a constant and reliable power transmission rolling mills performance and uptime rely on Universal cardan Joints which are the most critical equipment of the drivetrain. Fatigue assessment of the most important components of the Universal cardan Joint is therefore definitely vital for the mill in order to avoid failures and consequently downtimes. Considering the component's dimensions and weight and in addition the time needed in order to reproduce full scale tests, a new approach to study the Universal cardan joint's main components fatigue behavior has been investigated. It consists in reproducing the local stress condition of the main joint components on small scale specimens that can be tested by conventional laboratory fatigue machines. The aim of this study is to analyze the most critical stress concentration areas in the universal cardan joints highlighting how a universal cardan joint Wöhler curve can be determined through laboratory tests of small scale specimens.
\end{abstract}

Keywords: Danjoint; Universal cardan joint; Fatigue assessment; Drivetrain; Finite element model; Wöhler curve, Specimen case hardening.

Senior engineer - mechanics, Danieli \& C. Officine Meccaniche S.p.A., Danieli Service, Italy. Senior engineer - mechanics, Danieli \& C. Officine Meccaniche S.p.A., Danieli Service, Italy.

Vice President drivetrain technology, Danieli \& C. Officine Meccaniche S.p.A., Danieli Service, Italy.

4 Design Engineer Research and Development, Danieli \& C. Officine Meccaniche S.p.A., Danieli Research Center, Italy.

5 Vice President, Design Research and Development, Danieli \& C. Officine Meccaniche S.p.A., Danieli Research Center, Italy.

Senior Researcher, Montanuniversität Leoben, Chair of Mechanical Engineering, Austria.

Head of Chair, Montanuniversität Leoben, Chair of Mechanical Engineering, Austria.

Assistant professor, Università di Udine,Dip. Ingegneria Elettrica Gestionale Meccanica, Italy. 


\section{INTRODUCTION}

A fatigue verification procedure with the aim of accurately assess the lifetime of a component should be primarily based on fatigue tests results. The best condition represents full scale component tests, but in many cases costs, duration, different sizes, huge dimensions and/or local multiaxial stress conditions make it unaffordable. A way to overcome this issue is to perform fatigue tests by appropriate designed small scale specimens and transfer the results in order to achieve a local Wöhler curve valid for a specific critical point of the component. Local material, metallurgical properties, heat treatment parameters and stress distribution of the specimens must be the same as the area of the component which should be assessed. Since the specimens must represent properly the real local component features (i.e. microstructure, hardness etc.), the production method has to be accurately established. For an universal cardan joint the fatigue life assessment is not straightforward because the components assembled together have several influence factors, like different geometries, materials, heat treatments, and local stress gradients, which exhibit a significant effect on the fatigue behavior. The universal cardan joint limit torque calculated with international standard is too conservative compared to the real transmitted value due to the fact that some parameters are difficult to be evaluated without tests. Therefore, in this paper an experimental procedure is presented to assess the real alternating limit torque, that can be transmitted by the DANJOINT cardan spindle, and obtain the actual Wöhler curve of each cardan spindle's size. The next step is to investigate the best strategy to evaluate the pulsating limit torque based on the real alternating limit value and to optimize design, material and heat treatment parameters of each spindle's size to extend their expected fatigue life.

\section{MATERIAL AND METHODS}

\subsection{Universal Cardan Joint}

In rolling mills for the metal industry it is usual to transmit power between two parallel shafts not in the same straight line, thus cardan spindle are widely used to accommodate the angular misalignment resulting from the offset of the shafts. Cardan spindles are suitable for applications where the maximum angular misalignment required is higher than $2^{\circ}$ with a reachable limit of $20^{\circ}$. For rolling mills the spindle size ranges from $180 \mathrm{~mm}$ to $1.400 \mathrm{~mm}$ (up to date biggest size manufactured), with an overall length of the biggest spindle size of $14.100 \mathrm{~mm}$. All the parts of DANJOINT cardan spindle are made of high quality forgings or castings. The materials used are quenched \& tempered and case hardened steels. Cardan spindle are usually installed on:

\subsubsection{Hot strip mills edger and roughing stands}

- Steckel mills roughing and finishing stands;

- Bar, section and wire rod mills vertical and horizontal stands;

- Heavy section mills;

- Breakdown mills;

- Pipe mills;

- Hot levelers. 


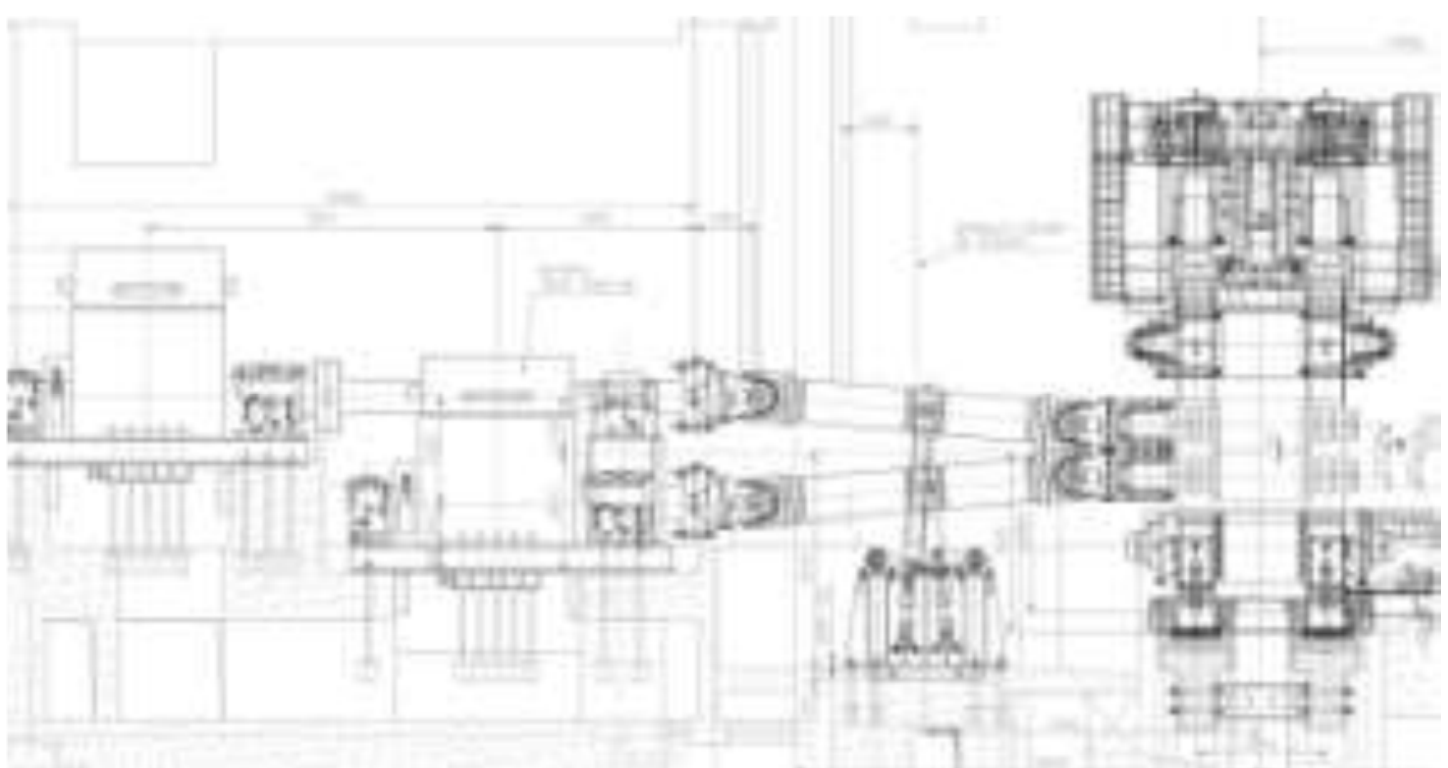

Figure 1: Mill stand cardan spindles arrangement

A cardan spindle consists of two universal cardan joints connected to each other through a central body. Two main versions are manufactured: fixed type and telescopic type with length compensation system to follow the position of work rolls during shifting. Both types can be designed for vertical or horizontal applications.

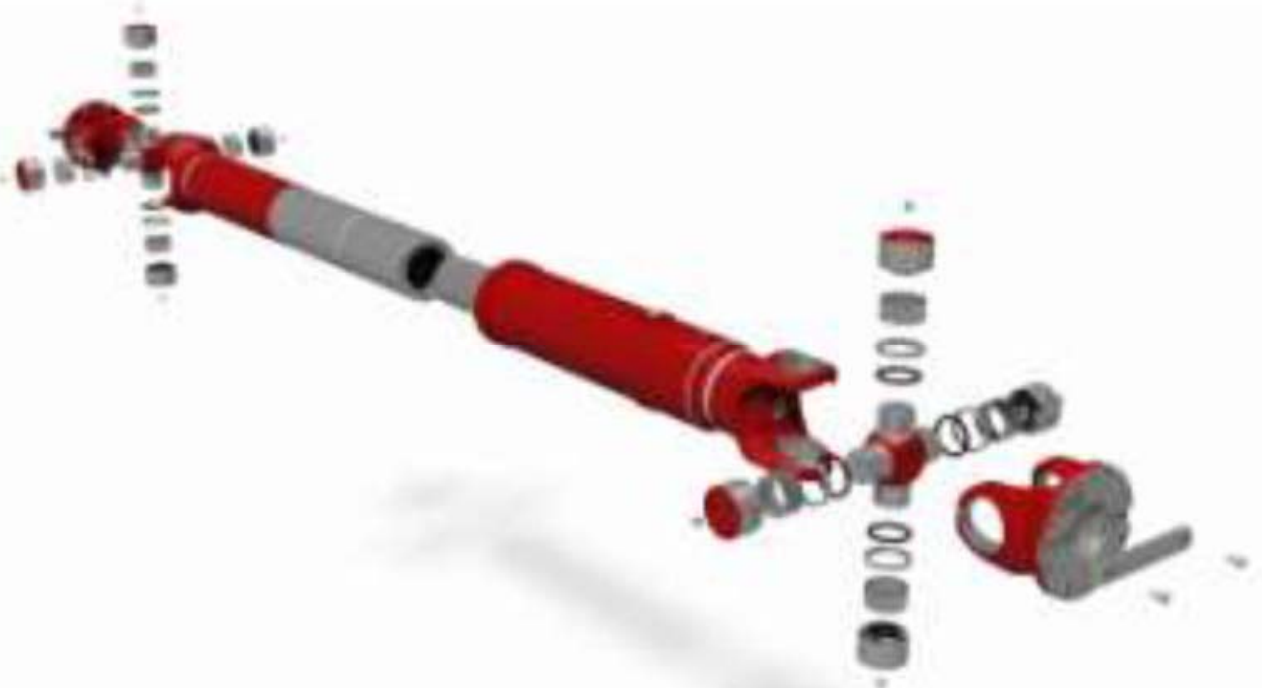

Figure 2: DANJOINT cardan spindle

Each universal cardan joint is made of two yokes, one cross and four bearings; its main feature is to allow the torque to be transmitted between two shafts inclined at angles to one another. While the driving shaft angular velocity $\omega 1$ is uniform, the driven shaft angular velocity $\omega 2$ varies harmonically with each revolution of the driving shaft. The amplitude of the sinusoidal variation of $\omega 2$ as well as the differential angle $\varphi(\alpha 1-\alpha 2)$ is largely dependent on the angle of deviation $\beta$. In a cardan spindle the input and output shaft are running synchronously and the spindle is working homokinetically[1]. 

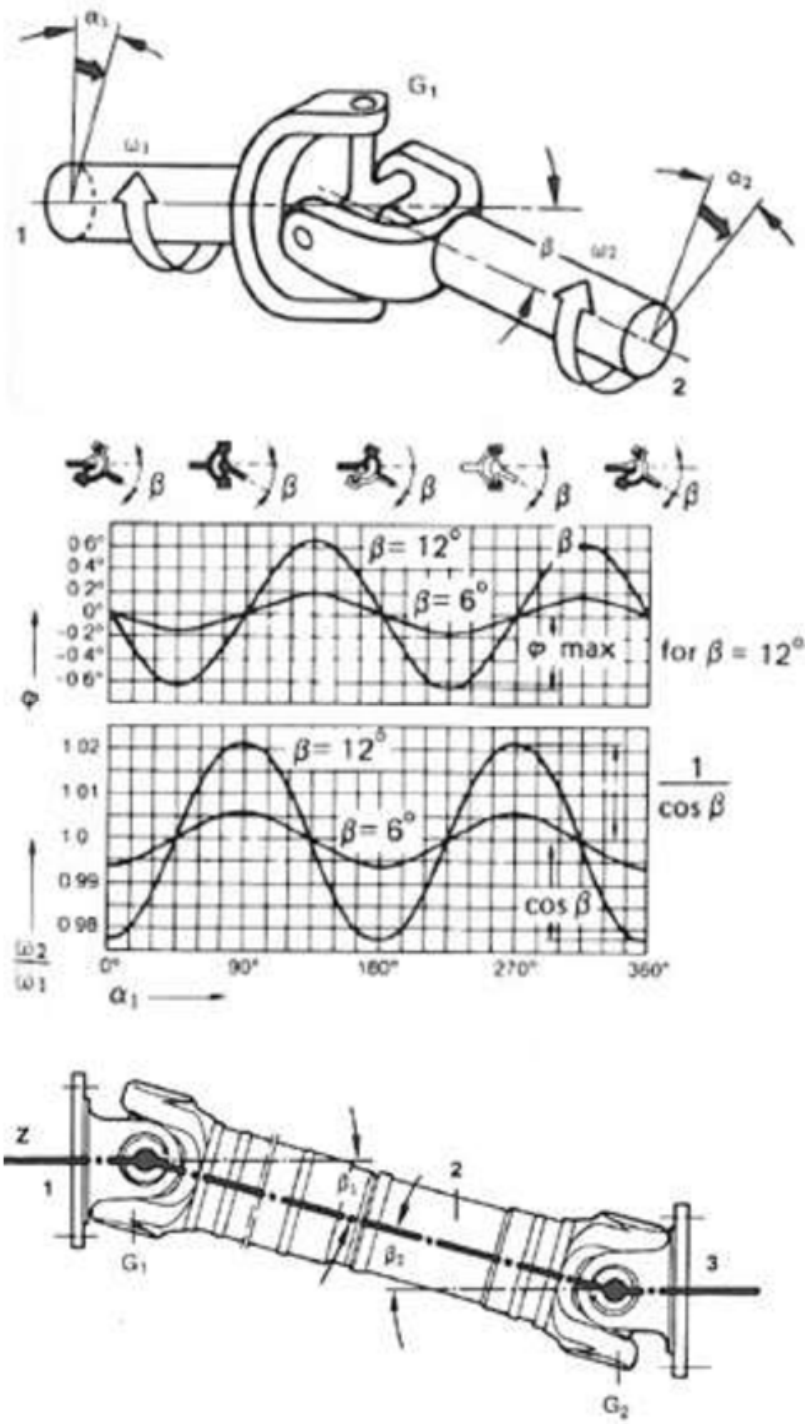

Figure 3: Kinematic of universal cardan joint [1]

The universal cardan joint classification is based on their size, that is the outer diameter of the joint, and each size is characterized by the following catalogue torques:

- Mk: limit steady torque (small unfrequent fluctuations allowed);

- Mdw: limit alternating reversible torque (2*10e6 cycles);

- Mdsch: limit pulsating torque $(2 * 10 \mathrm{e} 6$ cycles);

- Mcs: limit yielding material torque=1.3 Mk.

The selection of a universal cardan cardan joint is carried out by comparing the catalogue torques with the torque to be transmitted considering the nominal motor torque, the max rolling torque and the motor overloads. Based on the specific application, like roughing stand, finishing stand etc., the minimum acceptable safety factors are given by selection rules and past experiences on field.

\subsubsection{Full scale test rig}

The idea of building up a test machine to get the universal cardan joints Wöhler curve has been analyzed in terms of costs and time. A 3D-model of the machine has been designed in order to estimate the manufacturing, automation and personnel costs involved to run the tests. Thereby the time estimation includes the machine 
construction and the time needed to perform all the tests. Three different cardan spindle size have been considered to be tested, each size at several alternating torque levels. More than one cardan joint must be tested for each torque level in order to consider the data scatter. The resulting time to perform only the test is about two years.
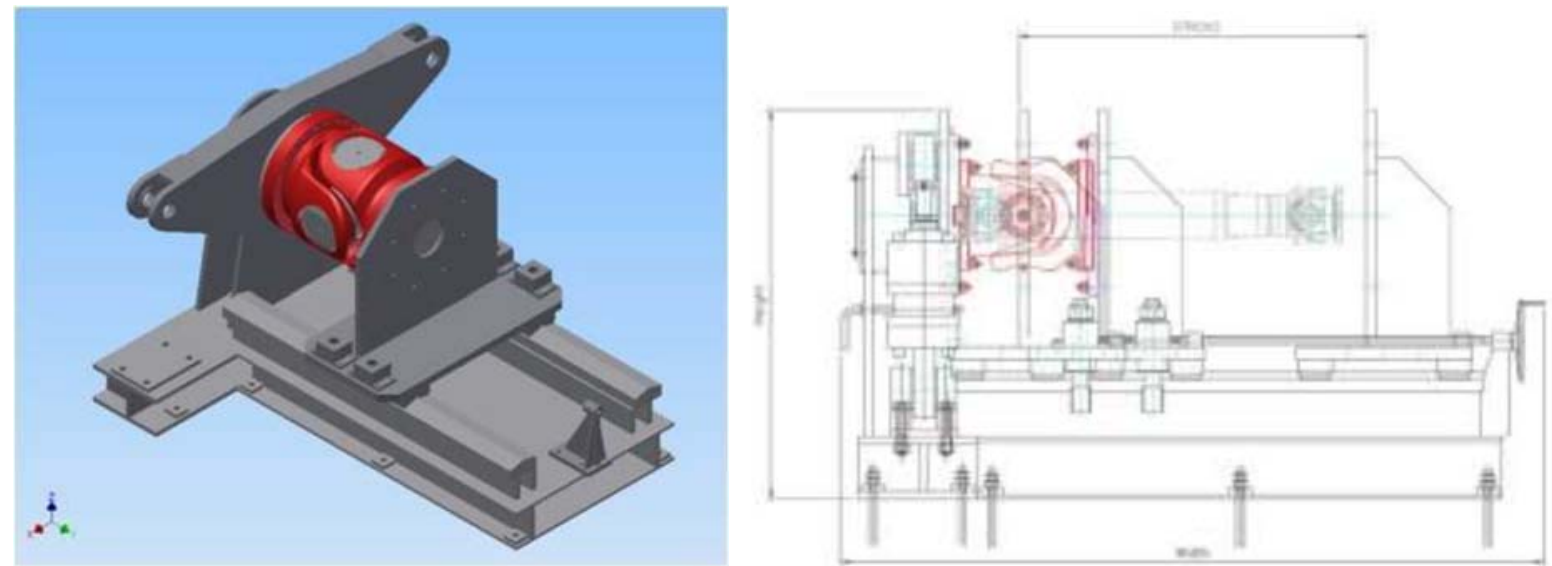

Figure 4: Full scale test rig.

Besides the high costs and time, the following aspects must be considered:

- Low results reliability due to several components involved during a single test;

- Difficulties to detect crack initiation properly;

- Uncertainty of failure of the same component during each test campaign which may lead to high scattering of the results;

- Data scatter increased by the replacement of one component or of the whole cardan joint after a failure occurs.

All the above considerations make the idea of a fatigue assessment only based on full scale tests unaffordable and lead to find a different experimental procedure. Anyway, the test machine can be used to validate the results of the experimental procedure by testing a complete universal cardan joint.

\subsubsection{Methodological approach based on small scale specimens}

The issue to obtain the real alternating limit torque that can be transmitted by the DANJOINT cardan spindle has been discussed with Montanuniversität Leoben (Austria), Chair of Mechanical Engineering, and University of Udine (Italy), Department of Electrical Management and Mechanical Engineering.

The following procedure has been defined based on their know-how about fatigue analyses and experimental test. Basically, the concept is to carry out fatigue tests on small scale specimens that replicate the main local features which influence the fatigue behavior of components. Subsequently, fatigue analyses of universal cardan joints are performed by means of a dedicated fatigue calculation software, whose parameters are calibrated by using the results of fatigue tests.

The starting point is the FEA of different sized universal cardan joints to find the fatigue critical point for each component. The analysis includes a comparison of the local stress condition in regard to the peak stress value, relative stress gradient and most stressed volume. Thereby the ratio between the CHD and the depth of the highstressed region for each spindle size is an important parameter and evaluated as well. 

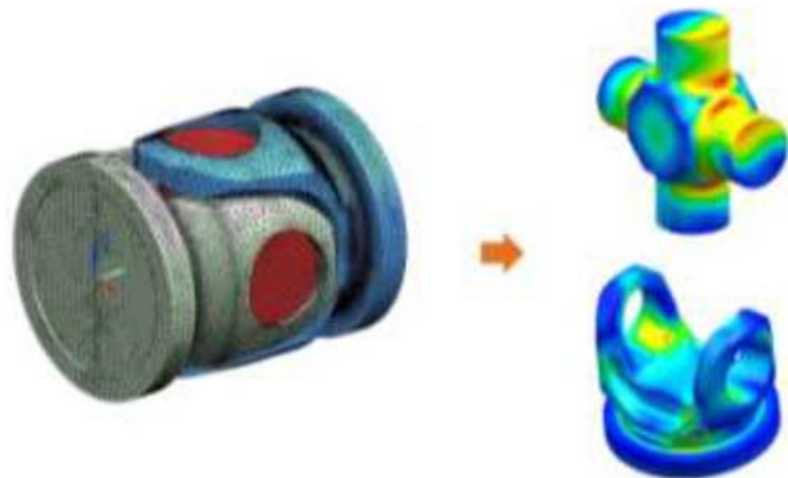

Figure 5: FEA of DANJOINT

Based on the FEA results, round specimen geometries are designed to consider the influence of different stress gradients.

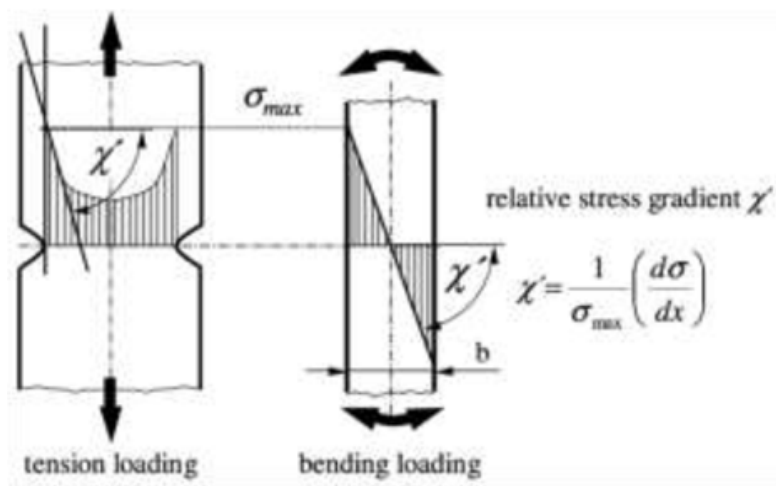

Figure 6: Evaluation of (relative) stress gradient [2]

Accompanying numerical analyses of specimen geometries are performed to achieve comparable local stress conditions as for the cardan joint. An evaluation of different $\mathrm{CHD}$ is carried out to ensure almost same ratios of the CHD to the high-stressed regions for the specimens as for the different cardan joint sizes.

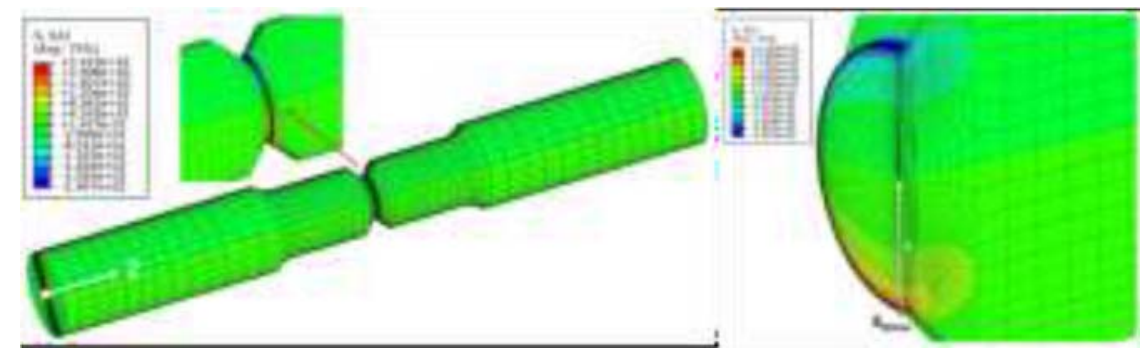

Figure 7: Illustration of small scale specimen geometry and local stress condition [3]

The specimen geometries are simple but the manufacturing process must be optimized to obtain the same properties of the real component in terms of metallurgical structure, heat treatment, core and surface hardness. To evaluate the $\mathrm{S} / \mathrm{N}$-curves of the designed round specimen geometries experimental fatigue tests for rotating bending and tension/compression at a stress ratio of $\mathrm{R}=-1$ are necessary. Based on these results it is possible to investigate the fatigue behavior of quenched \& tempered as well as case hardened components in regard to the ratio of the CHD to the depth of the high-stressed region. Accompanying measurements of local properties such as surface roughness, hardness measurements, fracture surface 
analysis, metallography and measurement of residual stresses are the basis for the transferability of the specimen results to the real component.
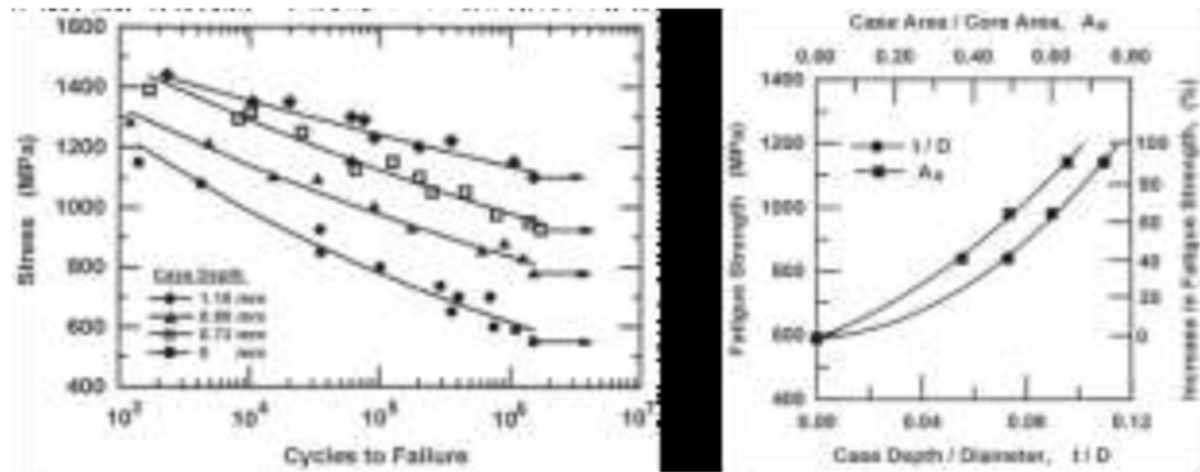

Figure 8: Exemplification of influence of CHD on fatigue strength [4].

After finishing the small scale specimen fatigue tests, the results will be implemented in the fatigue postprocessor FEMFAT [9]. One of the main parameters to be assessed is the Surface Treatment Factor that depends on the ratio of the CHD to the depth of the high-stressed region for a component and can be determined out of the experimental fatigue test results. In addition a comparison of the evaluated Surface Treatment Factors with default values proposed in the FKM-guidelines [10] will be performed. To validate this procedure the lifetime of the tested specimens will be again numerically assessed by the presented method and the final results are compared to the experimentally evaluated values.
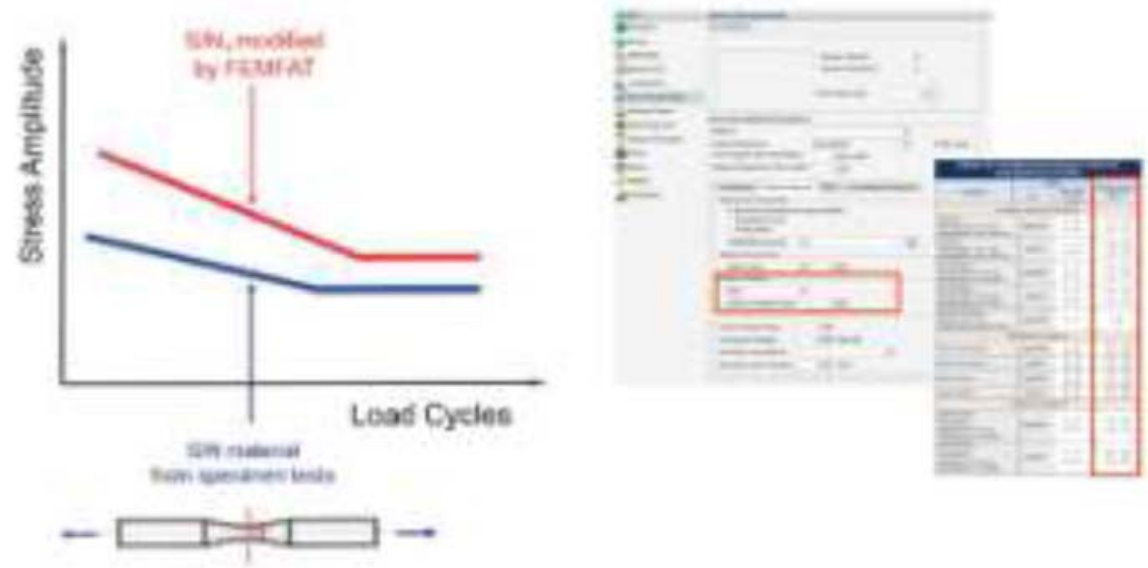

Figure 9: Surface Treatment Factor in FEMFAT

The final step is to set up a FEMFAT model of the universal cardan joint and carry out fatigue analyses on different spindle sizes.
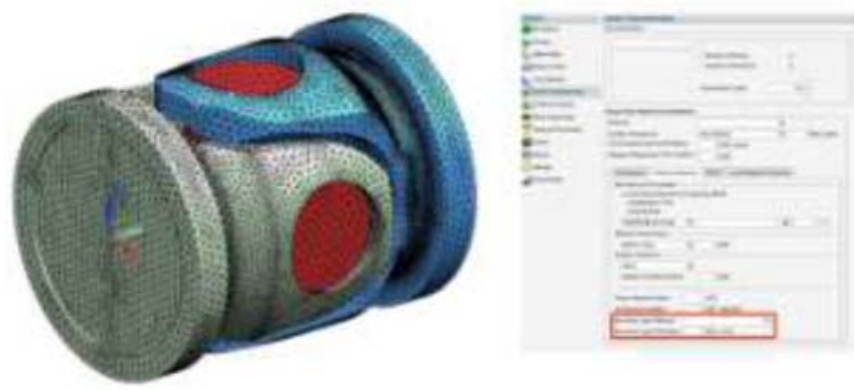

Figure 10: FEMFAT model of universal cardan joint 
The total amount of time to be involved is foreseen to be less than one year. By using all the information obtained with the experimental methodological approach referred above, the following targets can be achieved:

- Design, material and heat treatment optimization to extend the fatigue life;

- Analysis extended to all cardan spindle sizes and determination of limit torques with existing design and materials;

- Mechanical data acquisition of most common materials used in several application;

- Establish a technical/methodological know-how on fatigue calculation of mechanical components.

\subsubsection{Transfer of local properties to small scale specimen}

Each component of the universal cardan joint undergoes different local stress conditions and different fatigue failure mechanism. Moreover the components are characterized by different manufacturing processes and materials, so the experimental approach needs to be tuned for each component. The specimens designed for fatigue test must be as much as possible representative to the real component zone to be investigated in terms of metallographic characteristics. The best accurate way would be to cut out the specimen directly from the component but this would lead to high costs due to the huge number of specimens needed to be tested.

The forging process has influence on segregations and fiber orientation that must be reproduced with high accuracy in the specimens. Segregations are locations in the material matrix with different chemical composition that arise during solidification and hence, the fatigue behavior is strongly dependent on the cut out direction of specimen from the rough forged part.
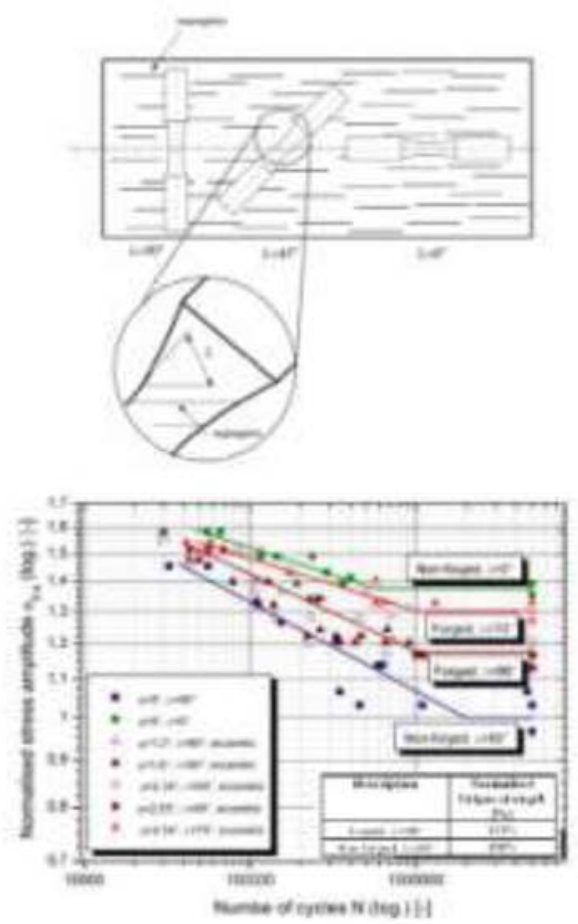

Figure 11: Influence of segregations on fatigue strength [5]

In order to understand which properties have to be transferred to the specimens, metallurgical analysis are executed on the real component. The first component to be 
investigated is the cross which is not subjected to contact stresses in the critical area. On the other hand, the fatigue life of yokes and bearings is primary limited by the local conditions in the contacted areas, but in these cases the presented procedure involving stress-free surface fatigue tests on small scale specimens is not applicable.

\subsubsection{Cross}

The FEA of the cross shows that the critical point is the fillet zone and the stress gradient is detected in the normal direction to the surface.
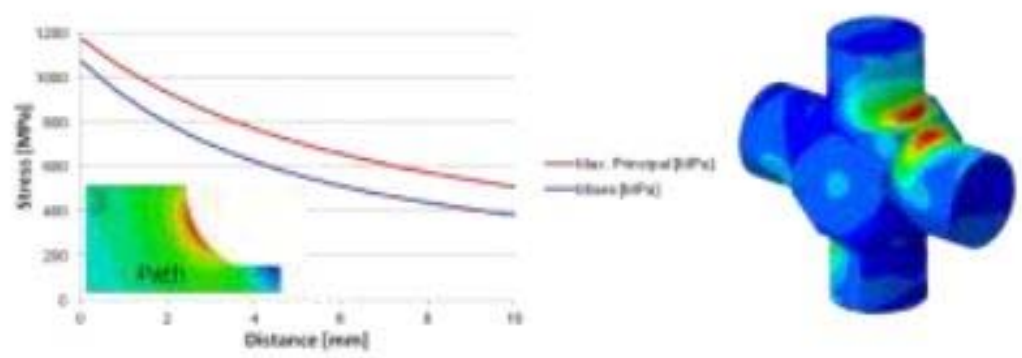

Figure 12: Results of FEA for cross.

Numerical analyses show a relatively shallow load stress distribution in depth. By comparing the stress distribution to the fatigue resistance variation in depth it is possible to estimate if the crack initiation point will be on the surface or in the core. The shallower the stress gradient, the most likely the crack will start at the core and therefore the hardened layer influence on the fatigue behavior seems to be negligible which can be verified by means of fatigue test.

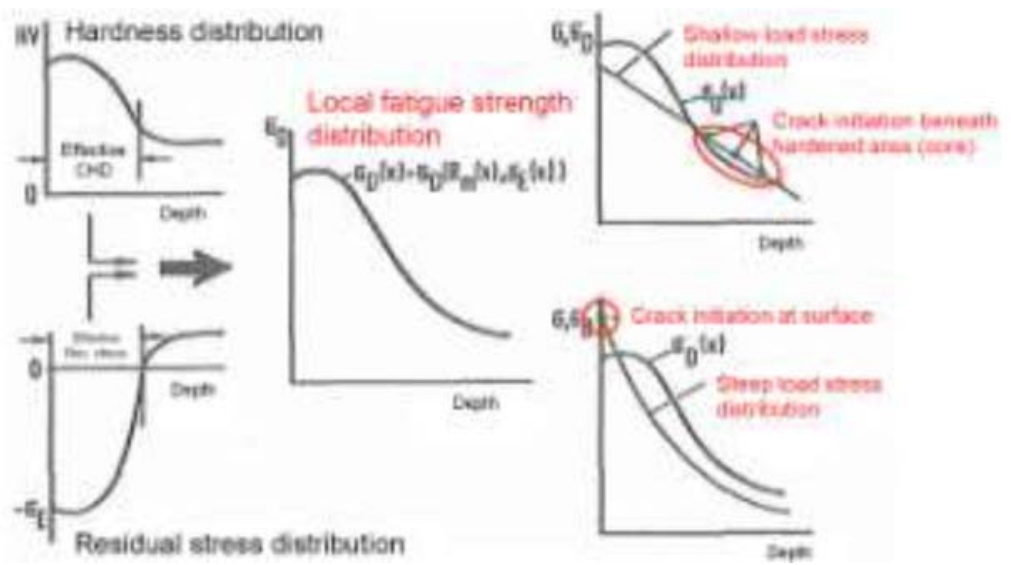

Figure 13: Method to assess crack initiation point according to [6]

In order to understand which are the properties at the fillet zone, the following metallurgical analyses have been performed on samples cut out from the fillet zone of a real finished cross:

- Macrography with acid etching for fiber orientation;

- Micrography for grain dimensions;

- Evaluation of Microinclusions;

- Vickers hardness test. 


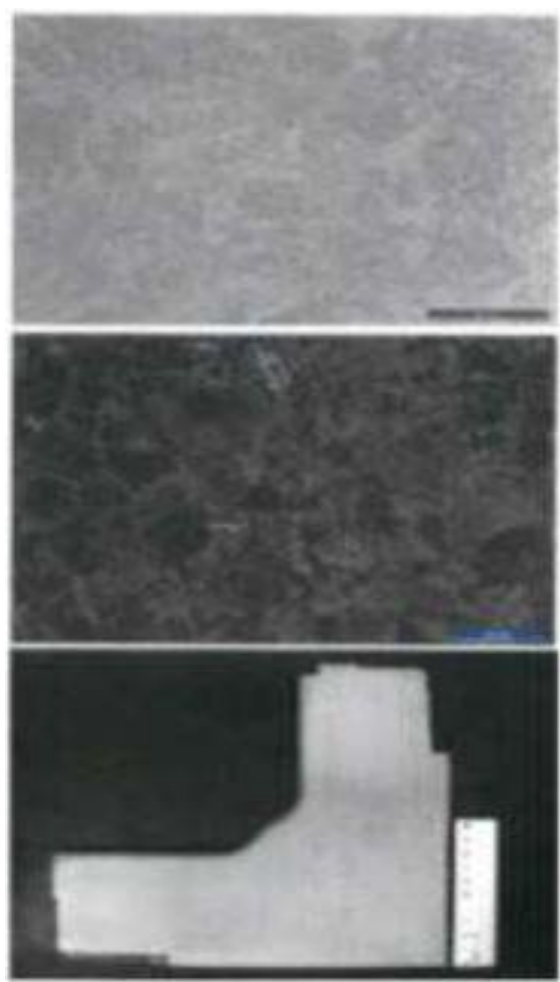

Figure 14: Metallographic analysis on a real finished cross.

The forging supplier suggested the proper forging process in order to make a rough forged part with the metallographic characteristics obtained from the above mentioned analysis. This part should be supplied as much as possible close to real component in terms of dimensions, forging procedure and quality controls. The aim is to cut out as many specimens as possible from one rough forging part to reduce costs and time. Before cutting out the specimens, a sample of the rough forged part will be analyzed to ensure that the metallographic properties will match with good accuracy the real cross ones. The specimens will be machined, heat treated and tested with the aim of:

1) Evaluation of $S / N$-curve of base material (no case hardening): The forged rough part have the same dimension and heat treatment of the existing cross and the specimen are cut out beneath the case hardened layer;

2) Investigation of the influence of case hardening on fatigue life: The specimens are cut out from a full annealed forged rough part. Two series of specimens are obtained: the first is case hardened, the second is quenched \& tempered;

3) Evaluation of $\mathrm{S} / \mathrm{N}$-curve of a quenched \& tempered steel and compare it to $\mathrm{S} / \mathrm{N}$ curve of base material (no case hardening): The forged rough part is made of quenched \& tempered steel without hardened layer.

\subsubsection{Fatigue assessment of yokes and bearings}

During normal operating conditions yokes and bearings undergo contact loads that result inmultiaxial local stress at the critical points. Therefore the transferability to common specimen fatigue tests with stress-free surfaces is not useful due to the different failure mechanisms, like contact fatigue and/or fretting. The fatigue issues on yokes and bearings can be attributed to fretting fatigue, a phenomenon induced by combined localized cyclic contact motion and external bulk fatigue loadings that may result in premature and dramatic failure of the contacting components. Crack nucleation and possibly crack propagation can be activated depending on fretting 
and fatigue load conditions. The two damage thresholds can be estimated by applying the Crossland high cycle fatigue criterion and the short crack arrest description respectively for crack nucleation and for the assessment of crack propagation [7].

Comparative fretting tests can be performed on dedicated small scale specimens but in general it is not possible to assess fretting fatigue in complex assemblies quantitatively only based on transferred results.
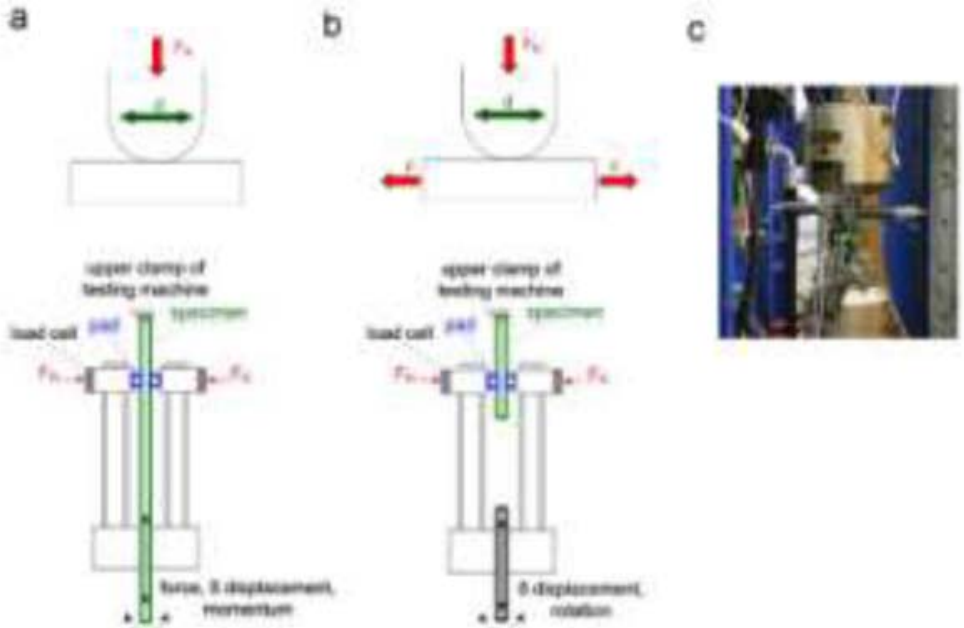

Figure 15: (a) fretting fatigue test set up, (b) fretting wear test set up, (c) mounted test rig [8]

During torque transmission the different amount of deformation of yokes and bearings causes a separation between their mating surfaces. The presence of vibrations during working may generate small amplitude oscillatory movement between contacting surfaces that, combined with cyclic bulk fatigue loading (the socalled fretting fatigue loading), can induce crack initiation which results in significant reduction of the cardan joint endurance.

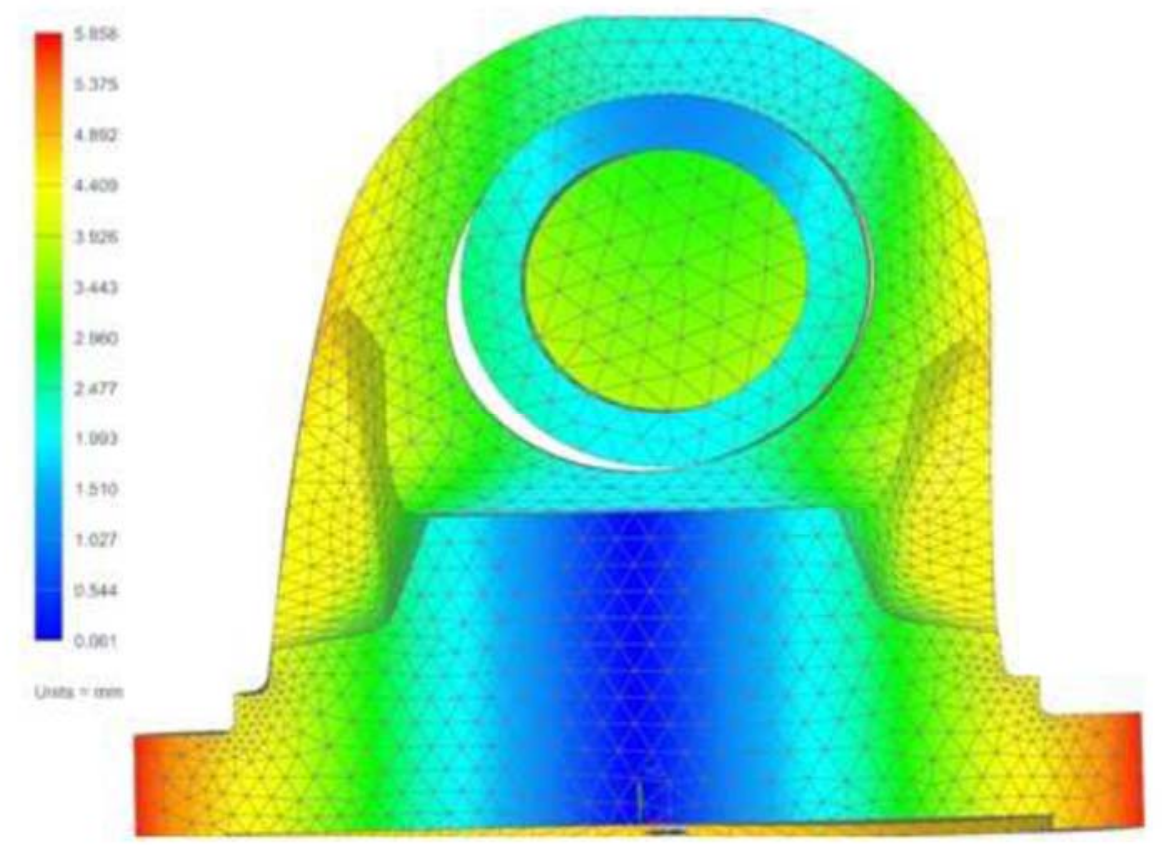

Figure 16: Yoke and bearing deformation. 
A transition fit between yokes and bearings is provided for ease of assembling/disassembling. With a press fit the separation of contact surface is slightly reduced at operating torques, so the benefits on fretting behavior are almost negligible but the assembling/disassembling procedure becomes more complicated. The following figure shows the pressure distribution on yoke's eye with an applied torque load. The increasing of diametrical interference from $0,088 \mathrm{~mm}$ to $0,2 \mathrm{~mm}$ is not leading to a reduction of the separation surface ( pressure equal to zero).

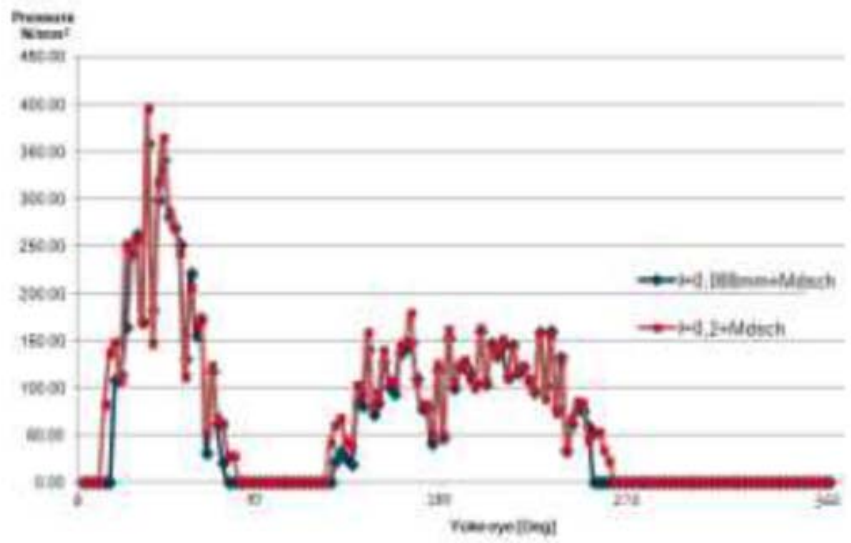

Figure 17: Contact pressure over yoke's eye.

A calculation procedure on contact fatigue by using a multiaxial fatigue method like Dang Van [11] or Crossland [12] is going to be defined in order to take into account in the endurance calculation of a component the multiaxial local stress condition induced by the contact. Possible solutions to improve fretting fatigue resistance are planned to be investigated:

- Optimization of the design of the whole assembly to increase the stiffness of yokes' eye thus reducing its deformation;

- Coatings to prevent wear and debris on contact surfaces;

- Deep rolling and shot peening to prevent fatigue crack propagation by introducing residual compression stress distribution.

\section{CONCLUSION}

An experimental methodology to study the fatigue behavior of universal cardan joints is presented. The procedure consists of designing small scale specimens that reproduce the local stress condition, material metallurgical properties and heat treatment of the main universal cardan joint's components. In this way the specimens are suitable to be tested by conventional laboratory fatigue test rig. The most critical stress concentration areas in the universal cardan joints' components are analyzed by means of FEA and their local Wöhler curves are then evaluated through fatigue laboratory tests. The universal cardan joints components' dimensions and weight makes a full scale test not affordable due to high time and costs needed, so the experimental methodology presented in this article is generally more applicable and useful. The fatigue assessment of the universal cardan joint provides the advantage to assess theendurance limit of the critical parts based on the working conditions of the specific application, with the aim of avoiding unexpected failures and consequent rolling mill downtimes. Moreover the methodology can be extended to calculate the fatigue behavior of other mechanical items. 


\section{ABBREVIATIONS}

\begin{tabular}{|l|l|}
\hline CHD & Case Hardening Depth \\
\hline FEA & Finite Element Analysis \\
\hline S/N & Stress/Number of cycles \\
\hline
\end{tabular}

\section{Acknowledgments}

Metallurgical analyses on real cross samples were conducted by LAB.MET. Metal Service Group Italy. The support by NUOVA CMF-Italy for definition of forging parts supply and specimen cut out is much appreciated. Special thanks to DANIELI Heat treatment, quality, and workshop departments for their continuous collaboration.

\section{REFERENCES}

1 Mancuso, J.R.: Couplings and Joints, Design, Selection and Application; Marcel Dekker, 1986;

2 Eichlseder W.: Fatigue analysis by local stress concept based on finite element results, Computers and Structures, Vol. 80, pp. 2109-2113, 2002;

3 Leitner M.: Life time increase and cost saving through evaluating the optimal case hardening depth for gears due to experimental and numerical investigations, Diploma thesis, Montanuniversität Leoben, 2009;

4 Genel K.; Demirkol M.: Effect of case depth on fatigue performance of AISI 8620, International Journal of Fatigue, Vol. 21, pp. 207-212, 1999;

5 Fröschl J.: Fatigue behaviour of forged components: Technological effects and multiaxial fatigue, Doctoral thesis, Montanuniversität Leoben, 2006;

$6 \quad$ Kloos, K. H.; Adelmann, J.; Bieker, G. Oppermann, T.: Oberflächen und Randschichteinflüsse auf die Schwingfestigkeitseigenschaften. VDIBerichte 661 (1988), 215-245;

7 Fouvry S., Kubiak K.: Development of a fretting.fatigue mapping concept: The effect of material properties and surface treatments, Wear267(2009) 2186-2199;

8 Christiner T. et al.:The fatigue endurance limit of a high strength $\mathrm{CrNi}$ steel in a fretting dominated regime, Tribology International, 2013;

9 FEMFAT, www.femfat.com;

10 Forschungskuratorium Maschinenbau: Rechnerischer Festigkeitsnachweis für Maschinenbauteile aus Stahl, Eisenguss- und Aluminiumwerkstoffen (FKM Guideline), 6. Auflage. Frankfurt am Main, VDMA-Verlag, 2012;

11 Dang Van K.: Fatigue analysis by the multiscale approach, High Cycle Metal Fatigue, from Theory to Applications, C.I.S.M. Courses and Lectures, No. 392, Springer Wien, New York, pp. 57-88, 1999;

12 CROSSLAND, B.: Effect of large hydrostatic pressure on the torsional fatigue strength of an alloy steel. In: Proc. Int. Conf. on Fatigue of Metals, Institution of Mechanical Engineers, London, 1956, pp. 138-149. 